\title{
A comparative assessment of Rapid Diagnosis Test (RDT) versus microscopy for malaria diagnosis in health care facilities
}

Theresia Njuabe Metoh ( $\nabla$ njuabe@yahoo.fr)

University of Bamenda https://orcid.org/0000-0003-3010-4380

Chi Tchampo Fru

University of Bamenda

Philip FonGah

University of Bamenda

Xiaonong Zhou

NIPD-CDC Shanghai, China

\section{Research}

Keywords: Malaria, Microscopy, Sensitivity, specificity, and HRP-2 RDT

Posted Date: January 7th, 2020

DOI: https://doi.org/10.21203/rs.2.20202/v1

License: (c) (i) This work is licensed under a Creative Commons Attribution 4.0 International License. Read Full License 


\section{Abstract}

Background: Management of malaria requires prompt diagnosis of malaria by microscopy, Rapid Diagnostic Tests (RDTs), or other available tools. The objective of this study was to determine the best approach on malaria diagnosis in detection of malaria parasite in the Northwest region, of Cameroon among different population groups.

Materials and method: The cross sectional study was conducted on 60 febrile patients who were directed to the laboratory department for blood screening at the Bamenda Regional Hospital after showing signs and symptoms of malaria infection. Blood sample were collected aseptically and dispensed into an EDTA container where RDTs and microscopic examination were performed to assess the presence of malaria parasites. Results from the rapid diagnostic kits were analyzed and compared to those obtained by general microscopy.

Results : Of the 60 samples enrolled 37 (61.7\%) were found to be positive with blood films examination while in rapid diagnostic test 27 (45\%) were positive. Based on frequency of infection by age 20-35 years had $13(35.1 \%)$ as the most vulnerable group, followed by 0 -5 year with $11(29.7 \%), 6-19$ had $6(16.2 \%)$ while age group of 36-59 had $5(16.0 \%)$ and lastly the age group of 60 and above had least value of 2 (5.4\%). The prevalence of malaria obtained through microscopy (62\%) was significantly higher than in RDT (45\%). Considering microscopy as the gold standard, RDT exhibited high specificity (100\%) and sensitivity (73\%) with positive predictive and negative predictive values of $100 \%$ and $70 \%$, respectively. The sensitivity of RDT increased significantly with increase in P. falciparum parasitaemia which was the plasmodium specie detected in all positive cases.

Conclusion: The study, therefore, highlights that the routine microscopy test demonstrated a superior sensitivity compared to RDT method of malaria diagnosis, however, RDT could be a useful tool in individuals suspected to show high degree of disease spectrum for quick intervention in order to avert danger associated with delayed diagnosis.

\section{Introduction}

Malaria has been known to mankind for thousands of years. Around 7,000-12,000 years ago with increase in temperatures in Africa, rise in humidity creating new water sources and the start of agriculture in the Middle East and North East Africa which brought about several water bodies and pools of water. This led to a favorable climate and an area for breeding and transmission of malaria parasites and its carrier, the mosquito [1] The World Health Organization (WHO) estimated that, 3.2 billion people are at risk of being infected with malaria and according to the latest estimates, 219 million cases of malaria occurred globally in 2017 and the disease led to 407,000 deaths [2]. Sub-Saharan Africa bears the highest burden of malaria, accounting for an estimated $90 \%$ of all malaria deaths worldwide of which more than $78 \%$ of malaria deaths occur in children aged $<5$ years. Over $90 \%$ Cameroonians are at risk of malaria infection, and about $41 \%$ have at least one episode of malaria each year [3]. The main strategy for 
malaria control as recommended by the WHO is a prompt and accurate diagnosis followed by effective treatment $[4,5]$. The confirmation of suspected malaria cases by microscopy or a rapid diagnostic test (RDT) before treatment [5] will result in a significant reduction of morbidity and mortality related to misdiagnosis. Although PCR may represent the best diagnostic method with high sensitivity and specificity [6], it is still costly and not very useful for routine diagnosis. Hence, microscopy and RDTs continue to be the primary choices for diagnosing malaria in most endemic countries. Microscopy is still considered the "gold standard" for malaria diagnosis in endemic countries because has a sensitivity of 50-500 parasites/ $\mu \mathrm{l}$ [7], is inexpensive, and allows the identification of species and parasite density [8,9], however the implementation of microscopy as diagnosis tool, especially in remote areas has been reported to be cumbersome and to requires trained personnel, microscopes and a source of electricity[10]. In remote areas of sub-Saharan Africa, RDTs have become the primary tool for the parasitological diagnosis or confirmation of malaria [11].The most widely used RDTs for malaria are based on the detection of parasite histidine-rich protein II (HRP2) and Plasmodium lactate dehydrogenase (pLDH) [12]. The major constraint of RDTs are false positives, because HRP2 persists in the blood for several days after infection clearance [13], and false negatives due to gene deletions [11]. Moreover, RDTs are thought to be more specific to P. falciparum infections [14]. Another drawback of RDTs is the occurrence of, falsenegative results. This situation may delay treatment process and increase the number of persons capable of infecting mosquitoes in the community. Variation of performance in the diagnosis method has been shown to have a great impact on individual treatment and epidemiological surveillance [15]., and some health professionals in endemic countries have lose confidence in test results and resolved to treat systematically all fever cases as malaria $[10,16]$., This may result the over- or under-diagnosis of malaria, with excessive use of anti-malarial drugs or negligent treatment, which invariably contributes to malaria morbidity and the development of resistance $[12,17]$. Clinical diagnosis is imprecise but remains the basis of therapeutic care for the majority of febrile patients in malaria endemic areas, where laboratory support is often out of reach. Scientific quantification or interpretation of the effects of malaria misdiagnosis on the treatment decision, epidemiologic records, or clinical studies has not been adequately investigated [18]. Despite an obvious need for improvement, malaria diagnosis is the most neglected area of malaria research, accounting for less than $0.25 \%(\$ 700,000)$ of the U.S.\$323 million investment in research and development in 2004 [18]. Rational therapy of malaria is essential to avoid non-target effects, to delay the advent of resistance, and to save cost on alternative drugs. Accurate diagnosis is the only way of effecting rational therapy. Confirmatory diagnosis before treatment initiation recently regained attention, partly influenced by the spread of drug resistance and thus the requirement of more expensive drugs unaffordable to resource-poor countries [19].. The objective of the present study was to assess the performance of RDT compare to microscopy, the two main techniques used in Cameroon for the diagnosis of malaria.

\section{Research Methodology \\ 2.1 Study area}


This study was conducted at the Regional hospital Bamenda located in the North West region found in Mezam division and in Mankon subdivision. It's a three-star high centered hospital created by the government for the entire population of the people of Bamenda. It incorporates a great number of patients a day coming from different areas of the region. Also having a qualified staff determined for the well-being of its patients

\subsection{Sample size and selection}

The cross-sectional study was conducted for 60 patients within Mezam division. A total of 60 patients consisting of 30 males and 30 females, varying of ages were enrolled from the 30th of Novembre to the 15th December 2016 at the regional hospital in Bamenda. These patients were admitted for malaria RDT and microscopy diagnosis after showing signs and symptoms of malaria infection. Patients were of a variety of age groups presented at medical consultation with signs and symptoms of malaria infection and confirmed by parasitological test.

\subsection{Sample collection}

Blood samples were collected by venipuncture into containers containing EDTA. After mixing, the blood samples were used to determine the hematocrit, for the preparation of thick and thin slides for microscopy, and for the RDTs assay.

\subsection{Microscopy analysis}

When making thick film, a drop of blood was placed at the center of the cream grease free slide and spread with the edge of another slide in a repeated coil shape to a diameter approximately $2 \mathrm{~cm}$. The slide was labelled and left horizontally for drying and are kept well to prevent them from dust and damage. It was then stained with $2 \mathrm{ml}$ of a $10 \%$ Giemsa solution and then washed after 10 min using clean water. As for the thin film, a drop of blood was placed at the center of the cream grease free slide the film is then spread using a smooth edged slide spreader. $2 \mathrm{ml}$ of methanol is applied on to it and allowed for fixation for a period of 2 minutes. After fixation $2 \mathrm{ml}$ of a $10 \%$ giemsa solution is then applied on the fixed smear and then washed after 10 min using clean water. A drop of immersion oil was applied on dried stained slides (thin and thick films) and examined microscopically for malaria parasites using 100X objective lens and result was recorded [20] .

The number of parasites per liter is calculated as;

[See supplementary files section to access the formula.]

\subsection{RDT Analysis}

Test device, buffer and specimen were allowed to equilibrate at room temperature $\left(10^{\circ} \mathrm{C}-30^{\circ} \mathrm{C}\right)$ prior to testing. The test cassette was removed from the foil pouch by tearing at the notch and then placed on level surface. $5 \mu$ l of whole blood were slowly added into the sample well. Then 3 drops of clearing buffer was added to the buffer well. As the test begins to work, a purple colour moving across the result window in the center of the test device. A result was read after 25 minutes. 


\subsection{Data Analysis}

Data was carefully entered in Excel spread sheet (version 2013) and analyzed using the statistical package for social sciences (SPSS) version 20 software. Proportions were calculated and the diagnostic performance was determined by calculating the test sensitivity, specificity, and predictive values.

\section{Results}

The prevalence of positive test in both microscopy and RDT diagnosis was highest (33.4\% and $35.1 \%$ respectively) among the 20 to 35 years age group (Fig. 1) and the least prevalence was seen in both diagnosis was seen in 60 and above age group (5.4\% in microscopy and $3.7 \%$ in RDT).

Figure 1: Prevalence of diagnosis with respect to age group

As regards to age, it was noticed that a greater proportion of getting positive patients with RDT diagnosis within the age ranges of 20 to 35 hence having patients of this age group most sensitive to RDT, by which may be due to the large number of patients diagnosed within this age group followed 0 to 5 age group 6 to 19,20 to 35 and 60 and above age groups respectively.

The overall prevalence of malaria parasites obtained through microscopy (62\%) was significantly higher than in RDT (45\%). Species of malaria parasite identified in all study participants was Plasmodium falciparum. The parasite density ranged from $40-400$ per $\mu \mathrm{L}$ of blood. The number of subjects with true/false negative and true/false positive RDT's results was presented in Table 1.

Table 1

No. of subjects with true/false negative and true/false positive RDT's diagnostic test results

\begin{tabular}{|c|c|c|c|}
\hline $\begin{array}{l}\text { TRUE } \\
\text { FALSE }\end{array}$ & POSITVE & NEGATIVE & TOTAL \\
\hline POSITIVE & 27 & 0 & 27 \\
\hline NEGATIVE & 10 & 23 & 33 \\
\hline TOTAL & 37 & 23 & 60 \\
\hline
\end{tabular}

From the study we have observe that RDT has a $73 \%$ of sensitivity. Hence RDT detects $73 \%$ of true ill patients, while $27 \%$ (false negative) will not be detected. Practically, this test detects $100 \%$ of malaria free patients. Implying that all patients diagnosed free of malaria parasites are actually healthy. PPV is $27 / 27$ $=1$ or $100 \%$ (Table 2). Hence all patients who test positive on RDT have the disease thereby indicating the presence of the plasmodium falciparum parasite in the infected patient. Also out of the 33 disease free 
patients, 23 patients test negative. This basically means that a person who test negative on RDT has a $23 / 33=0.70$ or a $70 \%$ likelihood of not having the disease.

Table 2

Specificity, Sensitivity PPV and NPV of RDT and microscopy diagnosis.

\begin{tabular}{|lllll|}
\hline METHOD & SPECIFICITY & SENSITIVITY & PPV & NPV \\
\hline RDT & 100 & $73 \%$ & 100 & $70 \%$ \\
\hline MICROSCOPY & 100 & 100 & 100 & 100 \\
\hline
\end{tabular}

In this study routine microscopic examination of stained blood films which is considered as the gold standard for malaria diagnosis had a sensitivity of $100 \%$ and was able to detect more parasites than the RDT (sensitivity $73 \%$ ). Though the specificity of microscopy and that of RDT was $100 \%$, microscopy has a high sensitivity and has the ability to quantify parasitemia which is a good advantage.

The sensitivity of RDT is seen to be highest in age group of 20 to $35(53 \%)$ having number of parasites ranging from 60 to 2720 (Table 3), followed by 6 to 19 (27\%) with parasites ranging from 65 to 1200,36 to $59(10.5 \%)$ with parasites ranging from 68 to 720,0 to $5(6.6 \%)$ with parasites ranging from 128 to 2600 and least in age group of 60 and above (2.9\%) with parasites number ranging from 100 to 650 .

Table 3

RDT sensitivity with respect to parasite density per age group.

\begin{tabular}{|lll|}
\hline Age group & Parasite density per $\mu$ l of blood & Sensitivity \\
\hline 0 to 5 & $128-2600$ & $6.6 \%$ \\
\hline 6 to 19 & $65-1200$ & $27 \%$ \\
\hline 20 to 35 & $60-2720$ & $53 \%$ \\
\hline 36 to 59 & $68-720$ & $10.5 \%$ \\
\hline 60 and above & $100-650$ & $2.9 \%$ \\
\hline
\end{tabular}

The optimal function of the immune system is mostly between the age ranges of 20 to 35 years, hence been able to detect the least amount of antigen in the system hence a great sensitivity in RDT. Whereas infants (ranging from 0 to 5 years) which have a developing immune system is not highly sensitive to antigens in the system hence a low sensitivity in RDT. The elderly (60 and above) have a suppressed immune system due to aging leads to less sensitivity of the test.

\section{Discussion}


Microscopy and RDT, recommended by WHO, revealed to be the most reliable tools for prompt and accurate diagnosis prior to an effective treatment of malaria suspected cases [5]. The sensitivity reported in this study does not attain the $95 \%$ recommended World Health Organization value [21]. This low sensitivity is disadvantageous as it will impair control intervention since a fraction of the infected population will be left untreated especially if RDT is the only available diagnostic tool. This could have important implications in health, transmission, and possibly mortality. The sensitivity of RDT reported in this study is lower than previous reported in Nigeria [22], Zambia, Zanzibar and Thailand [20, 23, 24]. Importantly, this study recorded the specificity of $100 \%$ for RDT which is far higher than the one reported in Burkina Faso[25], but similar to the observation made among the Nigerian isolates [26]. The difficulties and challenges faced by current RDTs have been reported showing a considerably varying specificities, sensitivities, numbers of false positives, numbers of false negatives and temperature tolerances of these tests [27]. False negative cases in this study were recorded for RDT using microscopy as diagnosis tool of reference.. The high proportion of false negative test resulted in the low sensitivity recorded in this study. This false negative could be explained by the effect of PfHRP2 sequence variation on the binding of specific mAbs [11, 28, 29, 30, 31]. In contrast, this sequence variation of PfHRP2 DNA obtained from isolates from African and South American was not found to be linked to varying sensitivities of RDTs [32]. Moreover, a prozone effect revealed to occur in PfHRP2 RDT and not with pLDH or aldolase based RDTs $[33,34]$. The storage temperature the RDT product by the marketers could also explain the observed low sensitivity. Several factors, including exposure to high temperature, storage and transportation conditions and training of health workers may likely influence test performance [25, 27, 35]. Interestingly, our study showed no false positive case and the absence of false positive test in this study is a characteristic of a good test. This is interesting because accurate diagnosis is the basis for an adequate disease control and for delaying the emergence and spread of antimalarial drugs resistance [15]. However, the rheumatoid factor cross-reacting in the blood could generate a false positive test line but this issue is now resolved by developing new products in which $\lg G$ is being replaced by $\lg M$ [36]. Also, cross-reactivity with heterophile antibodies may be another cause of false positive test [37]. False positives have been recorded due to infection with Schistosoma mekongi [38]. False positives are also caused by the persistence of PfHRP2 in the blood for long periods after parasite clearance[7, 39, 40].

Overall, the use of microscopy in diagnosis of malarial infection in adult population that comprises pregnant women can pose some challenges due to placental sequestration of parasites thus reducing the sensitivity of microscopy. However, the detection of peripheral blood HRP-2 genes is possible with malaria parasites, making RDT very useful in such condition [41]. The ability to detect placental infection by antigen detection when microscopy does not identify parasitaemia could have a significant impact on material and foetal health care. Besides, the difficulties associated with RDTs, such as genetic variability including polymorphisms in the PfHRP2 gene, and the persistence of antigens in the bloodstream following the elimination of parasites, highlights the importance for the development of alternative RDTs to the PfHRP2-based RDT. Several alternative malarial diagnostic targets have been identified and explored including heat-shock protein 70 , dihydrofolate reductase-thymidylate synthase, haemdetoxification protein, glutamate-rich protein, hypoxanthine phosphoribosyltransferase and 
phosphoglycerate mutase $[42,43]$. Again, a promising alternative to RDTs is the PCR $[6,15]$, a molecular technique that has proven to be the most sensitive and specific method available as it allows the detection of parasitemia as low as 2-5 parasites/ $\mu \mathrm{l}$ [3] However, it is expensive, complex, and not appropriate for field use; Again, it has to be validated by the WHO before its implementation in health facilities.

\section{Conclusions}

Stained blood film microscopy and rapid diagnostic test each with its characteristics, strength and the limitation together present the best hope for diagnosis as a key component of successful malaria control hence rapid diagnostic test does not eliminate the need for stained blood film malaria microscopy therefore all negative rapid diagnostic test must be followed with stained blood film microscopy to confirm the result. Microscopy is the more reliable method in areas where malaria is most prevalent. RDT offers a good alternative, being an easy and rapid method that does not require an experienced laboratory technician. However, there is a need for the development of alternative RDTs to the PfHRP2-based RDT. Therefore, while waiting for the implementation of these new techniques, when PfHRP2-based RDT is used alone, it should be followed with stained blood film microscopy to ascertain in the degree of infection and to know the malaria specie involved for proper treatment. The present study demonstrates that RDTs can act as a diagnostic tool to manage malaria in resource poor settings with limited access to expert microscopy as they are easy to use and perform.

\section{Declarations}

\section{Ethical Approval and consent to participate}

This study was approved by the Ethics Committees of the Ministry of Public Health Cameroon and was registered with controlled-trials.com in the website: https://clinicaltrials.gov/ct2/show/NCT02974348 under the registration number NCT02974348.

Participants provided written informed consent before enrollment in this study

\section{Consent for publication}

Not applicable

\section{Availability of data and material}

Data and material will be made available to the readers of malaria Journal since it is an open access Journal

\section{Competing Interests}

All authors declared no conflict of interest 


\section{Funding}

This study was sponsored in part sponsored by the Chinese National Science and Technology Project grant No. 2012ZX10004-220 and by the Ministry of Higher Education through annual small research grant offered by the minister to support research activities in government owned Universities

\section{Study Limitations}

The number of participants enrolled was determined by the short period of recruitment which is two weeks from the $15^{\text {th }}$ of April to the 30th of April 2016. The trend of the outcome might have a slight change with an increased number of participants.

\section{Authors' contributions}

MTN and ZXN designed and organized the study. CTF and PFG carried out the field and laboratory work. CTF and MTN wrote the manuscript. CTF and PFG analysed data and wrote the manuscript. All authors read and approved the final manuscript.

\section{Acknowledgements}

Our profound gratitude to all participants, patients and staff at the Regional hospital Bamenda located in the North West region found in Mezam division and in Mankon subdivision

\section{References}

1. Blanford Jl, Blanford S, Crane RG , Mann ME , Paaijmans KP , Schreiber KV , and Thomas MB. Implications of temperature variation for malaria parasite development across Africa. Sci Rep. 2013; 3: 1300.

2. World malaria report 2018. Geneva: World Health Organization; 2018.

3. Mbenda HGN, Awasthi G, Singh PK, Gouado I and Das A 2014 Does malaria epidemiology project Cameroon as 'Africa in miniature'? J. Biosci. 39 727-738] DOI 10.1007/s12038-014-9451-y

4. Gerstl S, Dunkley S, Mukhtar A, De Smet M, Baker S, Maikere J. Assessment of two malaria rapid diagnostic tests in children under five years of age, with follow-up of false-positive pLDH test results, in a hyperendemic falciparum malaria area, Sierra Leone. Malar J. 2010;9:28.

5. WHO. Guidelines for the treatment of malaria. Geneva: World Health Organization; 2010.

6. Zhou X, Huang J-L, Njuabe MT, Li S-G, Chen J-H \& Zhou X-N: A molecular survey of febrile cases in malaria endemic areas along China-Myanmar border in Yunnan province, People's Republic of China. Parasite, 2014, 21, 27.

7. Moody A. Rapid diagnostic test for malaria parasites. Clin Microbiol. 2002;15:66-78.

8. Feleke DG, Tarko S, Hadush H. Performance comparison of CareStartTM HRP"/pLDH combo rapid malaria test with light microscopy in north-western Tigray, Ethiopia: a cross-sectional study. BMC 
Infect Dis. 2017;17:399.

9. WHO. Parasitological confirmation of malaria diagnosis. Geneva: World Health Organization; 2009.

10. WHO. World malaria report 2016. Geneva: World Health Organization; 2016

11. Kozycki CT, Umulisa N, Rulisa S, Mwikarago I, Musabyimana JP, et al. False-negative malaria rapid diagnostic tests in Rwanda: impact of Plasmodium falciparum isolates lacking hrp2 and declining malaria transmission. Malar J. 2017;16:123.

12. Ugah UI, Alo MN, Owolabi JO, Okata-Nwali OD, Ekejindu IM, et al. Evaluation of the utility value of three diagnostic methods in the detection of malaria parasites in endemic area. Malar J. 2017;16:189.

13. Humar A, Ohrt C, Harrington MA, Pillai D, Kain KC. Parasight test compared with the polymerase chain reaction and microscopy for the diagnosis of Plasmodium falciparum malaria in travelers. Am J Trop Med Hyg. 1997;56:44-8.

14. Mathison BA, Pritt BS. Update on malaria diagnostics test utilization. J Clin Microbiol. 2017;55:2009-17.

15. Berzosa P , Lucio A de, Romay-Barja M , Herrador Z, González V, García L, Fernández-Martínez A, Santana-Morales M , Ncogo P , Valladares B , Riloha M \& Benito Comparison of three diagnostic methods (microscopy, RDT, and PCR) for the detection of malaria parasites in representative samples from Equatorial Guinea. Malaria Journa/ volume 17, Article number: 333 (2018). https://malariajournal.biomedcentral.com/articles/10.1186/s12936-018-2481-4

16. Mangham LJ, Cundill B, Achonduh OA, Ambebila JN, Lele AK, Metoh TN, Ndive SN, Ndong IC, Nguela RL, Nji AM, Orang-Ojong B,Wiseman V, Pamen-Ngako J, Mbacham WF: Malaria prevalence and treatment of Febrile patients at health facilities and medicineretailers in Cameroon. Trop Med Int Health 2011, 17:330-342.

17. Metoh Njuabe T, Tahar R, Same-Ekobo, Foumane Ngane V, Soula G, Basco L. K (2010). Molecular epidemiology of malaria in Cameroon XXIX. Characterisation of DHFR and Drug Resistance Markers and Efficacy of Sulfadoxine-Pyrimethamine Monotherapy in Children in Niete (HEVECAM). Sciences et medicine d'afrique/science and medicines in Africa VOL 2, (1), p146-152.

18. "MalariaFactsheetN94". March 2014. Retrieved 28 August2014.

19. Barnish G, Bates I, Iboro J (2004) Newer drug combinations for malaria.

20. Hopkins, H., L. Bebell, W. Kambale, C. Dokomajilar, P.J. Rosenthal and G. Dorsey, 2008. Rapid diagnostic tests for malaria at sites of varying transmission intensity in Uganda. J. Infect. Dis., 197: 510-518.

21. WHO, 2000. Malaria diagnosis new perspectives. Report of a Joint WHO/USAID Informal Consultation, October 25-27, 2000, Geneva.

22. Ajumobi, O., K. Sabitu, P. Nguku, J. Kwaga and G. Ntadom et al., 2015. Performance of an HRP-2 rapid diagnostic test in nigerian children less than 5 years of age. Am. J. Trop. Med. Hygiene, 92: 828-833. 
23. Msellem, M.I., A. Martensson, G. Rodllant, A. Bhattarai and J. Stromberg et al. 2009. Influence of rapid malaria diagnostic tests on treatment and health outcome in fever patients, Zanzibar-A crossover validation study. PLoS Med., Vol. 6. 10.1371/journal.pmed.1000070

24. Nicastri, E., N. Bevilacqua, M.S. Schepisi, M.G. Paglia and S. Meschi et al., 2009. Accuracy of malaria diagnosis by microscopy, rapid diagnostic test and PCR methods and evidence of antimalarial over prescription in non-severe febrile patients in two Tanzanian hospitals. Am. J. Trop. Med. Hygiene, 80: 712-717.

25. Kiemde F, Tahita MC, Bonko MdA, Mens PF, Tinto H, van Hensbroek MB and Schalli HDFH. Implementation of a malaria rapid diagnostic test in a rural setting of Nanoro, Burkina Faso: from expectation to reality. Malar J (2018) 17:316 https://doi.org/10.1186/s12936-018-2468-1

26. Dougnon 2015 Dougnon, T.V., H.S. Bankole, Y.M.G. Hounmanou, S. Echebiri, P. Atchade and J. Mohammed, 2015. Comparative study of malaria prevalence among travellers in Nigeria (West Africa) using slide microscopy and a rapid diagnosis test. J. Parasitol. Res., Vol. 2015. $10.1155 / 2015 / 108707$

27. Mouatcho JC and Dean GoldringMalaria rapid diagnostic tests: challenges and prospects. Journal of Medical Microbiology (2013), 62, 1491-1505 DOI 10.1099/jmm.0.052506-0

28. Lee, N., Baker, J., Andrews, K. T., Gatton, M. L., Bell, D., Cheng, Q. \&McCarthy, J. (2006a). Effect of sequence variation in Plasmodium falciparum histidine-rich protein 2 on binding of specific monoclonal antibodies: implications for rapid diagnostic tests for malaria. J Clin Microbiol 44, 2773-2778.

29. Houze' , S., Hubert, V., Le Pessec, G., Le Bras, J. \& Clain, J. (2011). Combined deletions of pfhrp2 and pfhrp3 genes result in Plasmodium falciparum malaria false-negative rapid diagnostic test. $J$ Clin Microbiol 49, 2694-2696.

30. Kumar, N., Pande, V., Bhatt, R. M., Shah, N. K., Mishra, N., Srivastava, B., Valecha, N. \& Anvikar, A. R. (2013). Genetic deletion of HRP2 and HRP3 in Indian Plasmodium falciparum population and false negative malaria rapid diagnostic test. Acta Trop 125, 119-121.

31. Berhane A, Russom M, Bahta I, Hagos F, Ghirmai M, Uqubay S. Rapid diagnostic tests to detect Plasmodium falciparum infections in Eritrea: an investigation of reported false negative RDT results. Malar J. 2017;16:105.

32. Baker, J., Ho, M.-F., Pelecanos, A., Gatton, M., Chen, N., Abdullah, S., Albertini, A., Ariey, F., Barnwell, J. \& other authors (2010). Global sequence variation in the histidine-rich proteins 2 and 3 of Plasmodium falciparum: implications for the performance of malaria rapid diagnostic tests. Malar J 9, 129.

33. Gillet, P., Mori, M., Van Esbroeck, M., Van den Ende, J. \& Jacobs, J.(2009). Assessment of the prozone effect in malaria rapid diagnostic tests. Malar J 8, 271.

34. Luchavez, J., Baker, J., Alcantara, S., Belizario, V., Jr, Cheng, Q., McCarthy, J. S. \& Bell, D. (2011). Laboratory demonstration of a prozone-like effect in HRP2-detecting malaria rapid diagnostic tests:implications for clinical management. Malar J 10, 286 
35. Chiodini, P. L., Bowers, K., Jorgensen, P., Barnwell, J. W., Grady, K. K., Luchavez, J., Moody, A. H., Cenizal, A. \& Bell, D. (2007). The heat stability of Plasmodium lactate dehydrogenase-based and histidine-rich protein 2-based malaria rapid diagnostic tests. Trans R Soc Trop Med Hyg 101, 331337

36. Maltha J, Gillet $P$, Jacobs J. 2013. Malaria rapid diagnostic tests in endemic settings. Microbiol. Infect.19:399-407. 10.1111/1469-0691.12151

37. Moody and Chiodini, 2002 Moody, A.H. and P.L. Chiodini, 2002. Non-microscopic method for malaria diagnosis using OptiMAL IT, a second-generation dipstick for malaria pLDH antigen detection. Br. J. Biomed. Sci., 59: 228-231.

38. Leshem, E., Keller, N., Guthman, D., Grossman, T., Solomon, M.,Marva, E. \& Schwartz, E. (2011).FalsepositivePlasmodiumfalciparumhistidine-rich protein 2 immunocapture assay results foracuteschistosomiasiscaused bySchistosoma mekongi.J Clin Microbiol49, 2331-2332.

39. Houze' , S., Boly, M. D., Le Bras, J., Deloron, P. \& Faucher, J. F. (2009). PfHRP2 and PfLDH antigen detection for monitoring the efficacy of artemisinin-based combination therapy (ACT) in the treatment of uncomplicated falciparum malaria. Malar J 8, 211.

40. Kyabayinze, D. J., Tibenderana, J. K., Nassali, M., Tumwine, L. K., Riches, C., Montague, M., Counihan, H., Hamade, P., Van Geertruyden, J. P. \& Meek, S. (2011). Placental Plasmodium falciparum malaria infection: operational accuracy of HRP2 rapid diagnostic tests in a malaria endemic setting. Malar $\mathrm{J}$ 10, 306.

41. Leke, R.F.G., R.R. Djokam, R. Mbu, R.J. Leke and J. Fogako et al., 1999. Detection of the Plasmodium falciparum antigen histidine-rich protein 2 in blood of pregnant women: Implications for diagnosing placental malaria. J. Clin. Microbiol, 37: 2992-2996.

42. Guirgis, B. S. S., Sa' e Cunha, C., Gomes, I., Cavadas, M., Silva, I., Doria, G., Blatch, G. L., Baptista, P. V., Pereira, E. \& other authors (2012). Gold nanoparticle-based fluorescence immunoassay for malaria antigen detection. Anal Bioanal Chem 402, 1019-1027.

43. Kattenberg, J. H., Versteeg, I., Migchelsen, S. J., Gonza'lez, I. J., Perkins, M. D., Mens, P. F. \& Schallig, H. D. F. H. (2012a). New developments in malaria diagnostics: monoclonal antibodies against Plasmodium dihydrofolate reductase-thymidylate synthase, heme detoxification protein and glutamate rich protein. MAbs 4, 120-126.

\section{Figures}




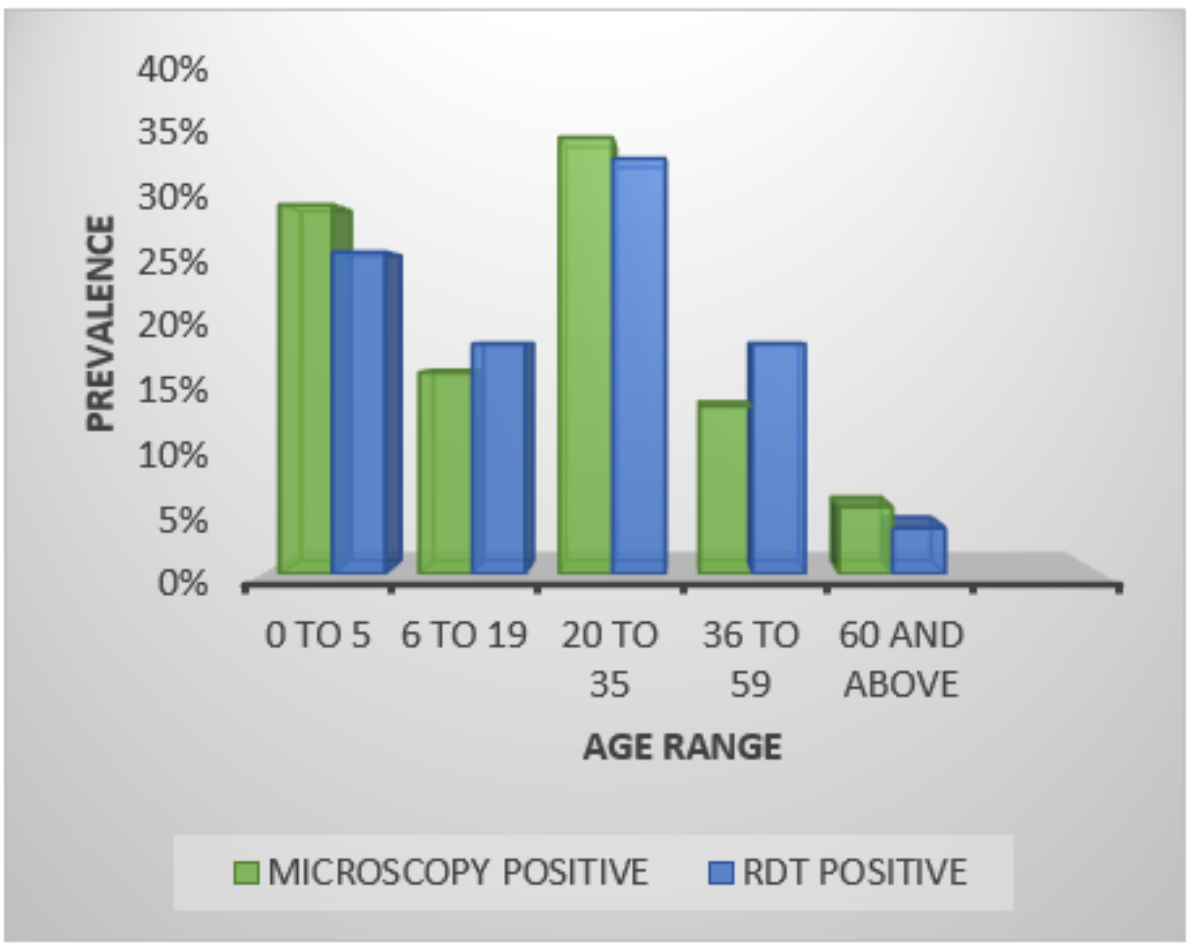

Figure 1

Frequency of diagnosis approach with respect to age group

\section{Supplementary Files}

This is a list of supplementary files associated with this preprint. Click to download.

- Formula.docx 